\title{
Reviewing Oncogenes and Proto-Oncogenes
}

\section{Nehal Batra ${ }^{1}$, Ishita Ghag' ${ }^{2}$ Karishma Babu ${ }^{3}$, Tejaswini Divanji ${ }^{4}$}

1 Jaypee Institute of Information and Technology

${ }^{2}$ SVKM Mithibai College

${ }^{3}$ SRM Institute of Science and Technology,

${ }^{4}$ Shobhaben Pratapbhai Patel School of Pharmacy and Technology Management

\section{ABSTRACT}

\section{Article Info}

Volume 8, Issue 3

Page Number : 458-479

Publication Issue

May-June-2021

Article History

Accepted : 25 May 2021

Published : 31 May 2021
This article is an examination of the Reviewing oncogenes and Protooncogenes The scientific development and subsequent "oncogenes and Proto-oncogenes" continues to influence the researchers all over the globe today. This article examines the research done and published by researchers and scientists. Consideration of current trends and data in scientific queries and demonstrates further aspects of this relationship. Additionally, this article explores options for oncogenes and Proto-oncogenes relationships.

Keywords : Oncogenes, Proto-oncogenes, Relationship, Researches, Current trends

\section{INTRODUCTION}

In 1865, Gregor Mendel discovered that a gene is a discrete unit of heredity in an organism. Genes are a region of DNA which encodes for a functional product. Humans have twenty-five thousand genes in genome. Cancer is a genetic disease that is caused by an accumulation of pernicious variation in the genome. An oncogene is a gene with a potential to cause cancer. In tumour cells, they are frequently mutated or expressed at high levels. Oncogenes are the result of mutation of proto-oncogenes. In 2018, there were 18.1 million new cases of cancer and 9.5 million cancer-related deaths all over the world. The cost of treatment of cancer is high and many people can't keep up with it. A study from 1984, discusses evidences of various mechanisms that are involved with the activation of proto-oncogene. In this paper, various aspects on relationship of proto-oncogenes and oncogenes is highlighted as a study is carried out from different select researches.

\section{METHODS}

The study was conducted using four databases Google Scholars SAGE, DOAJ and PubMed. Selection of papers were done based on keywords and theme relevant to this review. Further the published papers from these databases were arranged in systematic order with respect to year of publication.

\section{RESULTS AND DISCUSSION}

Genetic distribution of cancer in human chromosomes: 
Recombinant DNA technology has helped humanity to detect cancerous cells in our bodies. It is important to understand changes in DNA sequences to know how cancer development occurs. The first somatic mutation identified in human cancer was reported in a lineage of urinary bladder carcinoma cells as a change from glycine to valine in HRAS amino acid 12. To determine how cancer genes are distributed in chromosomes and whether certain chromosomes are primarily in the content of cancer genes, this study was undertaken. The conclusion of this study was that cancer genes were uniformly distributed across all human chromosomes growing together until the human genome evolved. While the number of mutations in one cell can number in the thousands especially in tumours harbouring alterations in DNA repair genes, the consensus is that most somatic point mutations in cancer genomes are passenger mutations and unlikely linked to mutations in other gene.

On being driven by other people's assertions that their chromosome of interest was richer in cancer genes, an analysis was done to have supported the result that the products of mutated genes promoted oncogenesis. Tier I had 572 genes which indicated the location of the cancer gene using the Catalogue Of Somatic Mutations In Cancer list (COSMIC). The genes in the Cancer gene Census had been divided into two tiers; where Tier I was considered. Information about the chromosome number, the starting and ending base pair, and the chromosome band, protein-coding genes, length of each base pair was noted (Genome Reference Consortium)

The Cancer gene census is still carrying on progressing with the cataloguing of the genes that contain mutations leading to cancer and how the altering of the gene sequences can lead to cancer. Utilizing a median-based method to identify outliers 4 potential outliers in chromosomes $13,17,19$, and 22 with chromosomes 13 having slightly fewer and chromosomes 17, 19, and 22 having slightly more cancer genes per base pair compared to the other 22 chromosomes. To conclude, Cancer genes were found to be evenly distributed across all human chromosomes. With respect to this, we can suggest that mutation in one gene can result in carcinogenesis. The physical separation of cancer genes implies any coordinate regulation must be transcriptional or epigenetic.

\section{Molecular cloning of a transforming gene from human bladder carcinoma cells}

Human cancer cell lines revealed the presence of controlling transformation genes. Carcinomas and sarcoma cell lines, high molecular weight DNA was isolated and two melanomas were used to transfect 3T3 fibroblastic mice. T24 (bladder) and A2182 (pulmonary carcinoma) and HT-1080 (fibrosarcoma cell line) were able to process 3T3 cells. Anchorindependent growth was observed in immunocompetent mice with no thymus gland and a first-cycle tumour transformant. They could transmit their malignant phenotypes in additional transfection cycles as they contained human DNA. When the southern blot analysis was performed on the T-24 transformants, it showed a single fragment of inherited DNA with the malignant phenotype suggesting that it had oncogene T24. When cloning human sequences with lambda Charon 9A (cloning vector), the resulting recombinant DNA molecule was shown to contain an EcoRI insert of 15 kilobases of human cell DNA. This resulted in the transformation of the 3T3 fibroblasts. Hence, the results were that molecular cloning of a transforming gene present in T24 human bladder carcinoma cells was successful.

The high molecular weight DNA which was isolated was tested for its ability to induce morphological transformation upon transfection with 3T3 cells. In order to find out the efficiency, K-HOS (a human cell line transformed by the Kirsten strain of murine sarcoma virus) was used. All transformants derived from K-HOS DNA tested showed a sarcoma virus that 
could be saved when they were superinfected with the murine leukaemia virus Moloney strain. The human tumour DNA's isolated from T24, A2182, and HT-1080 cells were the ones able to change the morphology of recipient 3T3 cells. T24 DNA exhibited the highest transforming activity (similar to K-HOS DNA). HT-1080 DNA was 5\%efficient and A2182 had an intermediate transforming efficiency. The morphological changes of 3T3 cells were highly refractile and overlaid one another in a random fashioned manner.

On investigating more about the properties of 3T3 cells, the growth efficiency was determined in a semisolid media. Transformants derived from each positive tumour cell line were found to form large colonies with an efficiency of $40 \%$ for HT-1080 and 95\% for T24 derived. To demonstrate that a human oncogene was the reason that the serial transmission of the transformed phenotype took place from human tumour cell lines to normal mouse cells, human marker DNA sequences were studied. The control didn't show any of the fragments thereby indicating that it was derived from transfecting human DNA. Due to the incorporation of large fraction of donor DNA by transfection competent 3T3 Cells, it hampered the identification of human DNA sequences harbouring the gene responsible for transformation. After few experiments were performed, it was suggested that the transforming genes of A2182 and HT-1060 human tumour cell lines must have a larger complexity than T24 carcinoma cells.

3T3 cells were further transfected with lambda T24 15A DNA, to determine its biological activity. Results were successful in demonstrating that a DNA fragment was cloned which contained an active transforming oncogene (present in T24 bladder carcinoma cells)

Certain dominant oncogenes are responsible for transmitting neoplastic properties from human tumour cell lines to normal cells. DNA isolated from tumour cell lines can transform mouse fibroblasts says recent studies yet this study shows us that DNA isolated from the bladder and pulmonary carcinoma was able to transmit malignant phenotype to $3 \mathrm{~T} 3$ cells. Such oncogenes are not restricted to cells derived from a certain type of neoplasia. Oncogenes present in different tumour cell lines have an independent genetic origin. The presence of certain repetitive sequences in human genomes has made it easier to detect DNA fragments with malignant transformation. Thereby, the experimental approaches have successfully allowed the molecular cloning of the transforming gene of human bladder (T24) carcinoma cells.

\section{Oncogenes: Clues to Carcinogenesis}

Late utilization of recombinant DNA strategies in malignancy research prompted the discovery of cell qualities with potential changing movement, called oncogenes(c-onc).

Oncogenes were first found and characterized in various retroviruses; these viral oncogenes (v-onc) are thought to have been gotten from the cell oncogenes (c-onc). By a combination of the $\mathrm{v}$-onc qualities into the host genome intense neoplastic change of the cell may happen. A few methods of oncogene enactment have examined that lead either to an expanded measurement of a quality item or to the arrangement of an adjusted quality item. The confinement of oncogenes in the human genome close to the breakpoints of explicit chromosome distortions engaged with different neoplasms like Burkitt lymphoma and a few leukaemia's accentuates the significance of these qualities in carcinogenesis.

Oncogenes are available in retroviruses as well as are homologous to DNA arrangements in ordinary, uninfected cells. By adding DNA groupings of this unfamiliar quality to their own DNA, these infections acquire changing movement. Transfer of purified, organically dynamic DNA sections to the beneficiary 
cells, named transfection, is ordinarily performed by the cultures to donor DNA by methods for calcium phosphate precipitation. It very well may be exhibited that DNA parts from an expansive range of various human tumour cell lines had the option to change nonneoplastic cells with high proficiency. DNA pieces confined from typical cells (for example human lung fibroblasts) needed to recognize capable changing movement. Results recommend that possibly changing qualities can be enacted as an outcome of DNA re-plans (for example separation of qualities from their ordinary administrative arrangements) and that numerous neoplasms contain enacted types of those qualities. The connections between chromosomal variations and human tumours on one hand and oncogenes on the other propose that DNA revisions may be a significant system for tumour enlistment by influencing the action of these qualities. Changes of oncogene DNA successions communicated at typical levels and expanded record of these qualities are two potential last makes driving a similar lamentable outcome. A paracrine creation of PDGF brings about the improvement of some connective tissue neoplasms. In actuality, high c-sister articulation has been found in different human tumour cell lines got from sarcoma and glioblastoma. Retroviruses can cause neoplasia either by transducing viral oncogenes fused into the viral genome from cell qualities during past rounds of contamination or by enacting occupant cell oncogenes by proximal combination.

The questions that were raised included whether the advancement of these neoplasms brought about by epigenetic changes or whether these tumours contain enacted genes that basically don't promptly change in the beneficiary (mouse) cells utilized in transfection tests.

Cells from patients experiencing a similar sort of tumour may contrast from one another on a subatomic premise (distinctive chromosomal breakpoints, various kinds of oncogenes included) may prompt new orders that will be of prognostic and remedial assistance to clinicians.

Late uses of monoclonal antibodies to malignant growth treatment have started fundamental endeavours to create antibodies against modified oncogene coded proteins and medications meddling with tyrosine phosphorylation. Regardless of whether these methodologies will turn out to be useful would be found later on.

\section{Amplification of cellular oncogenes in cancer cells}

Tumour-specific chromosomal translocation which imbalance the normal operation of a proto-oncogene can result into atypical expression of cellular oncogenes. Point mutations in oncogenes may lead to protein products with heightened tumorigenic potential. The amplification of cellular oncogenes can intensify their expression by increasing the amount of DNA template that will be available for mRNA production. Somatic amplification of specific genes has been shown in various responses of cells to stresses. Double minute chromosomes (dmins) and homogeneously staining chromosomal regions (HSR) associated with DNA amplification are two cytogenic abnormalities that were first discovered in tumour cells. During metaphase, dmins occur as small, spherical, paired chromosome like structure (centromere absent). Instead of alternate dark and light bands, HSRs stain as uniform with median intensity in trypsin-Giemsa-stained chromosome preparations. Both are rare in in-vivo tumour cells but as they are easily missed in cytogenetic analysis data is hard to find. Although, they are commonly found in malignant cells that are cultures (prominent in neuroblastoma cell lines). It has been observed that the culture conditions select tumour cells which have either dmins or HSRs and also that in cultured growth, dmins disappear with clonal population of cells with developed HSR, thereby suggesting these abnormalities are alternate forms of gene amplification. Dmins segregate unequally during mitosis and are many times lost from the nucleus as 
they lack centromeres. HSR have centromeres and are divided evenly in mitosis. Dmins and HSRs have been dominantly detected in tumour cells that are selected for cytotoxic drugs resistance, they are occasionally present in cancer cells before therapy. Amplification may range from a few-fold to several hundred-fold affecting at least 5 of 20 known cellular oncogenes. The first oncogene amplification was described involving the c-myc oncogene in a promyelocytic leukaemia cell line HL-607. The amplification was 832 times in both cell lines and cells taken from the patient. A correlation was discovered for c-myc amplification with dmins in a neuroendocrine cell line from a colon carcinoma COLO 320. An extensive search for changes in other oncogenes has unveiled amplifications that do not highlight as dmins or HSRs e.g., c-myb oncogene. This suggests that oncogene amplification maybe more regular than the structural alterations. In one case, an amplified oncogene has been detected in situ in its inhabitant chromosomal site that had an irregular banding region. Mutation does not appear to be essential for oncogene amplification. The occurrence of cells with amplified genes cytotoxic selection conditions, can differ by two orders of extent and is significantly augmented by existence of mitogenic substances (hormones or tumour promotors). Unusual replication occurs after inhibition of DNA synthesis and can lead to gene amplification with mitogenic hormones probably increasing inconsistent DNA replication. There are favoured chromosomal positions for amplification of cellular genes. Chromosomal rearrangements might enable gene amplification by positioning vital DNA sequences in a favourable array. Genetic instability is evidently boosted in cancer cells. some mutagenic insults are only carcinogenic as a result of subsequent amplification events facilitated by tumour promotors or by hormones. Amplified DNA in dmins must encompass an origin for DNA replication and must be nominated for in daughter cell populations, where it is disproportionately segregated. In 1 study, the length of an HSR was found to escalate during assortment of malignant cells for higher tumorigenicity. Amplified sequences in dmins and probably in HSRs as well, of tumours contain growthpromoting genes. Greater expression of an oncogene appears to be a requirement for the growth benefit of cells with extra copies of the gene. This could mainly contribute towards amplification of tumorigenesis. Tumour cell specificity of oncogene amplification has been found in three malignancies. The c-myc oncogene is amplified in maximum cases of the variant form of small-cell lung cancer, the presumed $\mathrm{N}$-myc oncogene is amplified in half of grade III and IV neuroblastomas and the c-erbB oncogene is frequently amplified in glioblastomas. Additionally, to dmins and HSRs, small-cell lung cancers and neuroblastomas often display a deletion of part of the short arm of chromosome 1 and chromosome 3. An activating mutation has been found in the neuroblastoma oncogene $\mathrm{N}$-ras. Amplification and enhanced expression of c-myc and N-myc seemingly occur during progression of human carcinoma of lung and neuroblastoma cells to an extra malignant phenotype. Amplification of an oncogene affects the malignant progression of now initiated cells. Amplified oncogenes may become transcriptionally silent on initiation of tumour cell differentiation. ${ }^{1}$

\section{The Role of Oncogenes in Chemical Carcinogenesis}

A number of oncogenes present in human tumours also as animal tumours are detected by the NIH/ 3T3 transfection assay. Studies in animal tumour model systems suggest that the chemicals or radiation may play a task within the activation of oncogenes by point mutation. Point mutations resulting in the activation of ras proto-oncogenes in several chemically induced rodent tumours are consistent with the known alkylation patterns of the carcinogens. The mutation at the 12th codon of the $\mathrm{H}$-ras gene detected in rat mammary tumours induced by methyl nitrosourea is consistent with the formation of the 06 methylguanine adduct. the aim mutation that activates the neu proto-oncogene in 
peripheral systema nervosum tumours in rats induced by ENU or MNU probably results from the binding of those potent genotoxic chemicals to DNA. The GGA-* GAA mutation observed within the 12th codon of ras oncogenes detected in MNU-induced mammary carcinomas is typically at the second guanine of this codon, albeit a uniform mutation at the primary guanine could also produce an activated ras oncogene. If the sequence of specificity for the binding of carcinogens to DNA corresponds to a known biological hot spot in an oncogene, then this chemical is often a really potent carcinogen. Several studies have shown that oncogene activation won't be the direct results of chemical interaction with DNA. it's possible in some instances that the chemical did activate the oncogene directly and is consistent with the chemical bonding to the DNA. In other instances, the chemical may have increased the background tumour incidence by a mechanism sort of a cytotoxicity or receptor-mediated promotion. If the pattern of activated oncogenes within the chemically induced tumours is different from that within the spontaneously occurring tumours, then the chemical probably caused the mutations during a number of the tumours. during a recent long-term carcinogenesis study, chronic exposure to tetranitromethane resulted during a high incidence of primary lung tumours in 344 rats and B6C3F1 mice. K-ras oncogenes with a GGT-GAT mutation within the 12th codon were observed in 18 of 19 rat lung tumours and 10 of 10 mouse lung tumours. The activation of the Kras oncogene in these TNMinduced lung tumours could even be the results of 1 or more actions of the chemical. it is a particular possibility that these activated $\mathrm{K}$-ras. Oncogenes with GC--AT transitions within the second base of the 12 th codon are spontaneous, but it is also possible that the irritant property of TNM could have promoted the cells, which activated the K-ras or enhanced the spontaneously occurring K-ras. Oncogene analysis on tumours from long-term carcinogenesis studies that are employed to assist identify potential human carcinogens are often useful in several ways. Lowdose and species-to-species extrapolation of risk from carcinogenic data may become more reliable from examination of oncogene activation and expression in animal model systems for carcinogenesis. ${ }^{2}$

\section{Oncogenes and cancer}

The finding of oncogenes rose from wide study of action of oncogenic animal retroviruses. Their genome has only three genes-gag, pol, and env; these three genes code for all the proteins required for replication. Via many genetic techniques, it was found that the transforming-cancer causing-ability of retroviruses was autonomous of the essential replicative genes. Oncogenic strains of virus frequently could not replicate suggesting that the oncogene swaps one of the three essential genes. The Rous sarcoma virus of chickens was the first retroviral genome to be characterised containing oncogene called src. A homologous gene was found in the DNA of normal non-malignant chicken cells. It was then, found in most vertebrates, as well as man. Alike results were found for other retroviral oncogenes. All were discovered in the normal cell genome, implying that viral oncogenes were derived from cellular genes that had been infected by virus before in evolution. A non-viral mechanism might convert proto-oncogenes into oncogenes in-situ. DNA can be inserted into normal cells from malignant cells, a process known as DNA transfection. The normal cells chosen are usually a mouse fibroblast cell line known as NIH3T3. Normal cells meaning that they respond to contact inhibition; monolayers of these cells on a Petri dish will grow to confluence. Some oncogenes after transfection into the NIH-3T3 cells will result in instant observable focal area of uncontrolled cell growth. It was reported that DNA extracted from human tumours could induce malignant transformation in NIH-3T3 cells, and normal tissue DNA cannot. The transforming element within the human tumour DNA was homologous to an oncogene, ras. Further research showed, three human genes are 
related to retroviral ras gene. The oncogenes in the animal retroviruses are related to normal cellular genes that are probably responsible for normal cell growth as they are found in normal tissue. MutationThe ras proto-oncogene can become oncogenic with a single point mutation, resultant in an amino acid substitution in the gene product. Chemical and physical carcinogens act by causing mutations at certain sites on cellular proto-oncogenes. An amplification-The c-myc oncogene is associated with acute leukaemia. Amplification may lead to overexpression of the gene product. $\mathrm{N}$-myc oncogene has also been associated with gene amplification in the late stages of metastasising neuroblastoma. Chromosomal translocation- translocation occurs steadily with particular tumours. In chronic myeloid leukaemia, there is the exact translocation of genes, including another oncogene, abl, from chromosome 9 to chromosome 22. Many studies show similarity between the oncogene products, the proteins encoded by the oncogene-and certain growth factors, growth factor receptors, and enzymes associated with the receptors like kinases. Normal cells produce growth factors. These factors act on target cells by binding to specific receptors on the cell surface. The different functions of oncogene products with expression and utilisation of growth factors propose a cascade of action: one oncogene may control the release of growth factors; another the availability of receptors, and a third an internal signal fired by the receptor. The correlation between oncogene expression and the clinical state of the patient or the grade of the tumour is still poor. Greater knowledge of the actions of these oncogenes will lead to specific antioncogene treatment. A monoclonal antibody or a drug designed to compete for the altered oncogene product without affecting the normal protein targeting the malignant cells will be good chemotherapy. ${ }^{3}$

\section{Oncogenes and gastrointestinal cancer}

There are known to be more than 40 oncogenes and proto-oncogenes and the function of their protein products falls mainly into three categories: 1) Protein kinases: Located on plasma membrane. As growth factors have protein kinases, there is a link between growth factors and oncogenes. 2) GTP- binding proteins: Oncogenes can bind to GTP.

3) Nuclear binding proteins: The c-myc oncogene product appears to be involved in replication of DNA and the fos gene product may influence the genesis of messenger

RNA.

Abnormalities in the expression of proto-oncogenes leads them to function as oncogenes and hence cause cancer in man. The potential role of abnormalities in oncogene expression in the development of gastrointestinal cancer has been intensively studied in recent years because these tumours are a cause of morbidity and mortality. The gastrointestinal tract a ready source of fresh tumour tissue as well as in colon, a lot of common and well-defined premalignant disorders are present. These include not only familial adenomatous polyposis, but also sporadic adenomatous polyps and longstanding ulcerative colitis. Most of early stats on oncogene expression in the gut came from work on tumour cell lines. Increased expression of fos, myc and ras oncogenes was found in a majority of colorectal carcinomas. Though there was no enhancement of the expression of 11 other oncogenes. 29 adenocarcinomas were studied and $72 \%$ of them had raised levels of c-myc. Another study stated that levels of myc expression were pointedly greater in tumours in the left colon compared with right sided lesions. Most tumours did show enhanced expression of c-myc, neither gene amplification nor rearrangement were seen in any case. One researcher reported amplification of c-myc in2 of 9 colonic tumours while another reported 2 in 32 colon cancers. Mutations of myb or ras genes in 19\% of colon cancers. $40 \%$ of colorectal cancers showed mutation of the ras gene and ras mutations were seen in the adenomatous and carcinomatous regions from five of the six tumours in which benign and malignant tumours coincided. It was noted that ras 
mutations in the same proportion of colonic cancers, and additionally in seven of eight villous adenomas. This raised the likelihood that activated ras genes contribute to the first stages of colonic tumour genesis in man. Another approach to the study of oncogene expression has been to research the expression of oncogene protein products. Raised amounts of the ras oncogene product (p21) were seen in 9 of 17 colonic carcinomas, with the most deeply invasive tumours showing normal p21 concentrations, metastases had lower levels. Immunohistology studies of p21 in sections of benign and malignant colonic tumours revealed that increased p21 concentrations were present only in the adenomas. These data are according to the hypothesis that ras expression may be a contributory think about early tumour formation. Upon comparison with normal colon and colon carcinomas, sporadic adenomatous polyps had the most intense staining of p62C-myc, particularly in areas of dysplastic change p62c myc is a nuclear associated protein, but exclusive cytoplasmic staining at the epithelial surface was characteristic of normal colonic biopsies. By contrast, adenomatous and malignant polyps from patients with familial adenomatous polyposis and biopsies of high-grade epithelial dysplasia and carcinoma from patients with ulcerative colitis, showed dominant nuclear staining at the mucosal surface. Carcinogenesis is a multistep process and the events leading to the formation of colorectal cancer are unlikely to be the result of the action of a single oncogene. It appears that ras proto-oncogene mutation could also be a crucial step in initiating formation of colonic tumours. ${ }^{4}$

\section{Oncogenes and Radiation Carcinogenesis}

Ionizing radiation is a well-established environmental carcinogen for humans and animals. The K-ras oncogene has been specifically implicated in other model systems of radiation-induced carcinogenesis. In $50 \%$ of rat skin carcinomas and $60 \%$ of rat thyroid tumours, activated K-ras genes were detected. K-ras is not the only specific target gene for ionizing radiation. Species and strain genetic factors are important in determining which oncogene will be part of the transformation pathway. It was found that in radiation-induced thymomas from $\mathrm{RF} / \mathrm{J}$ mice, both $\mathrm{K}$ and N-ras genes were mutated, while in C57B1/6J mice, these tumours contained mostly $\mathrm{N}$-ras and novel non-ras oncogenes. In canine leukaemia induced by exposure to gamma radiation from a cobalt source, the $\mathrm{N}$-ras oncogene was found to be activated. A murine osteosarcoma cell line derived from a 'Sr-induced tumour contained activated H-ras. In humans, ultraviolet radiation is an important environmental carcinogen, especially in rare DNA repair-deficiency syndromes such as xeroderma pigmentosum. In two tumours isolated from a single $\mathrm{XP}$ patient, the $\mathrm{N}$-ras oncogene was found to be mutationally activated. In addition, amplification of the c-myc and H-ras oncogenes was detected. The mutational activation of the $\mathrm{N}$-ras gene was a rare event, since tumours from seven other patients revealed no mutations in ras genes. But 6 of 10 tumours, exhibit amplified Hras. Another laboratory has reported that a melanoma cell line established from an XP patient also contained an $\mathrm{N}$-ras oncogene activated by the codon 61-point mutation. In a survey of human skin squamous cell carcinomas excised from sun-exposed body sites, it was reported that four of the eight tumours examined exhibited activated $\mathrm{H}$-ras, and two contained novel non-ras genes. point mutational activation of ras genes is only one of several types of oncogene alterations seen in radiation-induced transformation. Overexpression and amplification of the c-myc gene was also seen in a number of mice tumour types induced by ionizing radiation. A number of studies have been published dealing with effects of radiation on oncogenes in cultured cells. Ultraviolet radiation, along with other DNA-damaging agents, was found to induce c-fos expression in Chinese hamster ovary cells. In mouse C3H1OT 1/2 cells, UV-induced transformant showed a decreased level of c-fos expression, while c-myc 
expression was elevated. The killing of cancer cells by radiation is a well-known and important therapeutic modality. Also well-known is the phenomenon of resistance to radiation-induced cell killing that often occurs during progression of malignant tumours. Several laboratories have obtained evidence that cellular oncogenes are involved in the development of the radioresistant phenotype. A radiation-resistant human laryngeal cancer cell line was found to contain an activated raf oncogene. A laboratory found a raf gene to be involved in the transforming and radio resistance activities of DNA from a human skin cell line. Different results have been reported in a study that showed enhanced radiation resistance in NIH 3T3 cells transformed by transfection with ras oncogenes. Transfection of ras and myc also conferred an increase in radio resistance to primary fibroblast cells. One report showing increased sensitivity of C3H1OT $1 / 2$ cells to transformation by ionizing radiation after transfection with a c-myc gene (39) also suggests an early role for c-myc expression in radiation-induced cancer. Tumours were induced by exposure to radiation to male rats. Analyses of tumour RNA revealed enhanced cmyc expression in amplification-positive tumours. Frequent activation of the c-myc oncogene by amplification and rearrangements consistent with the known clastogenic effects of ionizing radiation on target DNA was seen. It can be concluded that c-myc oncogene amplification is not a frequent early event in radiation induced rat skin carcinogenesis. Amplification of specific genes in cancer is probably a random event. A huge difference observed in myc amplification between radiation-induced skin carcinomas and sarcomas indicates that in rat skin, as in several other animal models, tumour induction by a single etiological agent often involves the participation of different molecular pathways in different tissue types. A recent report from a laboratory showed that mouse thymomas induced by neutron irradiation exhibited a different pattern of oncogene activation than that seen with gamma radiation, including a unique ras-activating point mutation at codon 146. A clearer understanding of the molecular mechanisms of radiation carcinogenesis is required. 5

\section{Oncogenes in melanoma}

Genetic and epigenetic alterations form rock bottom for unstable neoplastic transformation characteristic. In melanocytic neoplasms, ultraviolet could even be a main environmental carcinogen. Also, germline mutations in tumour suppressor INK4A and oncogene CDK4 are identified in inherited melanoma. Epigenetic alterations like promoter methylation or protein degradation, are considered suppressive or dominant events involved in tumorigenesis and tumour progression. Target genes involved in cellular transformation and tumour progression are divided into two categories: proto-oncogenes and tumour suppressor genes. Tumour suppressor genes inactivation occurs mostly through an allelic deletion tailed by some extent mutation of the contralateral allele. Alterations in proto-oncogenes and tumour suppressor genes appear similarly predominant amongst human cancers. Some oncogenes have point mutations which modify encoded protein function. A genetic translocation positions an active promoter region next to a protooncogene. the normal control mechanisms are undermined, the oncogene is continually expressed, and cellular proliferation occurs. The breakpoints of a translocation happen within introns of two genes. After fusing, one frame is established with genetic information during a fusion, chimeric protein with oncogenic potential. If such a fusion protein is under the control of a lively promoter, then the oncogenic protein is synthesized. an area of the fusion protein, tyrosine-protein kinase activity is endlessly present, the signal transduction pathway is turned on, and cell proliferation persists. Overproduction of oncogenic proteins often occurs when there's an amplification of a specific chromosome region. When the amplification process generates a gaggle of repeated DNA segments 
confined to a neighbourhood of a chromosome, a homogeneous staining region, is produced. After DNA staining, these extrachromosomal DNA components have double minutes. As they're doing not have centromeres, DMs are randomly spread to daughter cells. In human tumours, $95 \%$ of cases of amplification are DMs and 5\% are HSRs. Amplification of oncogenes occurs during a kind of cancers, including melanoma. The proto-oncogenes when activated become tumour-specific oncogenes. The functions of proto-oncogenes are often classified as: (1) growth factors and their receptors (2) signal transducers (3) nuclear protooncogenes. Overexpression of certain protein $\mathrm{s}$ like basic fibroblast protein, platelet-derived protein, and epidermal protein are reported to be involved in melanoma as autocrine and paracrine loops in conjunction with their corresponding receptors. additionally, activating point mutations in protein receptors have also been observed. RET and MET mutations end in abnormal tyrosine kinase activity of the receptor protein. Oncogenes of the RAS family RAS-1, K -RAS-2, and N - RAS) each encodes a 21$\mathrm{kDa}$ protein, which shares homology with G-proteins. Oncogenic Ras proteins have sustained mutations that render them constitutively active by maintaining the proteins within the GTP-bound activated state. Activating RAS mutations are frequent in human cancers. Nuclear proto-oncogenes include members of the MYC family. Amplification of myc genes has been found during a kind of tumour types. Studies conducted by some researchers revealed that $24 \%$ of cultured malignant melanomas had activated RAS genes, with N-RAS being activated 10 times as frequently as Harvey -RAS. However, only 5- 6\% of nonculture primary and metastatic melanomas were found to contain mutated N-RAS. it had been reported N-RAS mutations in 33\% of primary and $26 \%$ of metastatic clinical melanoma samples. Prior it had been observed that $15 \%$ of cutaneous melanomas carry mutated N-RAS alleles. Mutations affecting the serine/threonine kinase B-RAF are reported in several tumours types, with high rates of melanoma. during a cohort of galvanic cell lines, two activating B-RAF point mutations cause increased kinase activity in $66 \%$ of melanoma samples. the primary B-RAF mutation found in melanoma was the V599E substitution, which occurred within the absence of RAS mutations, suggesting that it had been sufficient to deregulate this pathway. A recent study analysed tissues of melanoma and melanocytic nevi for mutations in BRAF exon 15 In 41/60 metastases, 4/5 primary melanomas, and 63/77 nevi, they observed mutations resulting in the V599E substitution. Among the downstream effectors of the RAS/MAPK signalling cascade, Cyclin D1 could even be a crucial regulator of the G1/S cell cycle transition. In melanoma, amplification of Cyclin D1 has been identified mostly in acral melanomas. Extra c-MYC oncogene copies are reported in 4 out of 8 primary and 11 out of 33 melanoma metastases. In melanoma, overexpression of HDM2 has been observed within the absence of amplification. Overexpression of HDM2 correlates with improved clinical outcomes in melanoma patients, independent of tumour thickness, the foremost important prognostic marker in localized disease. The recent implementation of highthroughput technologies can identify candidate oncogenes and tumour suppressor genes not before linked in melanoma. An observation is that alterations of Wnt signalling pathway are associated with human melanoma progression. One characteristic of melanocyte transformation is that these cells obtain the facility to form their own growth factors to form autocrine stimulatory loops. The three MGSA/GRO genes alpha, beta, and gamma affect melanoma. Blocking the binding of MGSA/GRO to melanoma cells causes growth inhibition (overcome by a quite MGSA/GRO). The protein products of oncogenes include cytokines and growth factors, protein receptors, and proteins involved in signalling pathways that promote cell division. In melanoma, BCL-2 is reported to be overexpressed in primary and metastatic lesions. This 
phenotype is claimed to tumour progression. Inhibition of this gene in vitro is linked to deregulation of apoptosis and increased sensitivity to cytotoxic drugs in melanoma cells. ${ }^{6}$

\section{MicroRNAs as oncogenes}

MicroRNAs (miRNAs) are non-coding RNAs that function as endogenic initiations of the RNA interference pathway. Anomalous expression of miRNA genes could end in human diseases, like cancer. The deletion at chromosome 13q14 has been studied for long because of its high frequency in chronic lymphocytic lymphoma and various solid tumours. the foremost probable contender for the cancer susceptibility gene at this site is that the miRNA cluster mir-15a-16, which contains, mir-15a and mir-16-1. Recent data suggested that miR-15a also as miR-16 potentiate the normal apoptotic response by targeting the anti-apoptotic gene BCL-2. miRNAs work as tumour suppressors, and reexpression of miR-15a-16 in lymphoma cells that harbour the 13q14 deletion promotes apoptosis. Chromosome 13q31 region is intensified in follicular lymphoma, B-cell lymphoma and various lungs, head and neck carcinomas. Chromosome 13, ORF 25 is strongly correlated with disease. Elevated expression of the mature miRNAs from this cluster, together represented as mir-17-92, has been proved in primary lymphoma and an in-depth range of tumour-derived cell lines. The expression levels of 217 miRNA genes across 334 primary tumours, normal tissues and tumour-derived cell lines were examined using multicolour flow-sorting. Tumours displayed an expression profile implicational that within the tissues from which they were resultant. The widespread reduction in miRNA levels might indicate the reduced differentiation that's a property of cancer. Histology demonstrates that tumours display the poorest degree of differentiation with rock bottom levels of miRNA expression. Though most miRNA genes display reduced expression in cancer, there are notable exemptions just like the mir-17-92 cluster.
The unpremeditated role of these miRNAs in cancer during a mouse model for lymphoma was displayed. Expression of oncogene c-Myc is directed from the immunoglobulin heavy chain enhancer (Em-Myc) leading to ectopic expression in B-lymphocyte progenitor cells and thereby, promotion of B-cell lymphoma. The co-expression of miR-17-19b which is a curtailed portion of miR-17-92, strongly accelerated lymphomagenesis. This was the first functional evidence of a miRNA, or any noncoding RNA, acting as a mammalian oncogene. The cellular function of miR-17-92 is unknown, the pathology of tumours indicates low rates of apoptosis. The key function of miR-17-92 appears to revoke Myc induced apoptosis. A confirmed target of miR-17 and miR-20 is that the transcript of the cell cycle transcription factor gene E2F1. Directed deletion of E2F1 doesn't accelerate Em-Myc lymphomagenesis. miR-19 has been established to downregulate the tumour suppressor PTEN. An unconnected miRNA gene, mir-155, might function as an oncogene in lymphoma. High expression of mir-155 and its host gene, BIC, has been reported in several kinds of B-cell lymphoma. This miRNA endorses cell proliferation. One model for tumorigenesis proposes that a touch fraction of cells during a tumour have properties of stem cells and are responsible for initiating and maintaining the tumour. Tumours retain expression of vegetative cell miRNAs, including miR-17-92. ${ }^{7}$

\section{Kras as a key oncogene and therapeutic target in pancreatic cancer}

The association of mutant Kras with carcinoma was established decades ago. Recent tumour genome sequencing studies have established the prevalence of mutant Kras in Pancreatic Intraepithelial Neoplasia (PanINs), the foremost common precursor lesion, and in carcinoma with greater precision. Results from the above studies confirmed the notion that over $90 \%$ of early-stage PanIN, as well as invasive tumours, express mutant Kras. Insight into the functional role of oncogenic Kras during the onset of carcinoma has 
been obtained using genetically engineered mouse models of the disease. KC mice express oncogenic Kras from the earliest stages of pancreatic embryonic development but have normal pancreas at birth and progress in grade over time. KC mice provided the first line of evidence that mutant Kras was necessary and sufficient for the initiation of carcinoma. The observation that Kras mutations occur at how higher frequency than carcinoma in humans is recapitulated in mouse studies, where-although every single pancreatic vegetative cell expresses mutant Kras from the primary pancreas development-PanIN lesions occur sporadically and only several weeks after birth. it's possible that even low, subclinical levels of local or systemic inflammation might promote the formation of PanINs, in presence of mutant Kras. In mice that activate the expression of the Kras mutant within the adult pancreas, carcinogenesis only occurs upon induction of chronic or acute pancreatitis. Even in KC mice, the induction of acute pancreatitis results in rapid and extensive PanIN formation. Human carcinoma cell lines are extensively used to study the disease. Knock-down studies have pinpointed Krasdependent and independent human cell lines and verfied a Kras "signature." Amplification of not only Kras, but also upregulation of genes involved in cell survival also as epithelial differentiation are key characteristics found within the Kras-dependency signature and are predictive of Kras "addiction". Different morphology and expression of ductal genes vs. mesenchymal-lineage genes differed in their dependence on oncogenic Kras. within the iKras model, oncogenic Kras is expressed in an inducible, tissue-specific and reversible manner. oncogenic Kras could be turned off at different stages of carcinogenesis and thus the results studied. Kras inactivation in PanINs resulted in rapid tissue remodelling: the PanIN cells re-differentiated into acinar cells, and thus the desmoplastic stroma was cleared through a thus far not fully understood mechanism. Kras inactivation in advanced PanINs led to massive vegetative cell death, in conjunction with some redifferentiation of acinar cells that then became proliferative and partially repopulated the pancreas parenchyma. a uniform effect was seen with Kras inactivation in tumours. an extra study including metastatic carcinoma (Collins et al., 2012b) and in vivo imaging showed regression of primary tumours and metastases. However, a subset of the tumour cells survived during a dormant state but could resume rapid growth upon Kras re-activation. Primary tumour cell lines derived from iKras mice carrying a mutant allele of p53 were Kras-independent for his or her growth in two-dimensional cell culture but required Kras for three-dimensional growth. Kras appears to induce the switch between a mostly aerobic metabolism, characteristic of the healthy pancreas, with an anaerobic mechanism mainly through the acid pathway, which is claimed to cancer cells. Additionally, it's also been shown that Kras regulates glutamine metabolism through noncanonical methods to assist within the maintenance of the tumour cell's redox state. oncogenic Kras promotes tumorigenesis by inducing expression of NRF2, a key component within the reactive oxygen species detoxification program, which reducing ROS levels is important for PanIN/cancer progression. Thus, if the ROS detoxification program is compromised because it occurs in mice lacking NRF2 expression, then pancreatic carcinogenesis is inhibited, indicating that this is often a fundamental mechanism to permit cells to bypass early barriers to carcinogenesis. Kras regulates other key cellular functions that are associated with the elevated energy needs of cancer: Macropinocytosis, induced by oncogenic Kras, allows the cancer cells to accumulate albumin from the encompassing extracellular space, and use it to supply Krebs cycle intermediates. In iKras* mice, inactivation of oncogenic Kras at any stage of carcinogenesis results in loss of proliferation also as smooth muscle actin expression within the stroma. The recent swell of inhibitors that prevent Kras activity indirectly is extremely exciting and promising. These inhibitors leave the traditional post- 
translational modification of the oncoprotein, Removing the necessity for alternative processing, but prevent its activity by interrupting its localization to the membrane or block the required interactions with its activating proteins. The MAPK pathway comprises of a kinase cascade, by which Raf kinases are activated by Kras and, activate MEK1/2. MEK kinases phosphorylate alongside ERK1/2 MAPK signalling is active in PanIN lesions also as in late-stage carcinoma, both in human tumours and in mice. Since both MAPK and PI3K signalling is active during a sizable amount of tumour types, small-molecule inhibitors for every pathway are developed. The MAPK pathway are often obstructed at the extent of Raf. the utilization of Raf inhibitors in Kras mutant tumours leads to the paradoxical upregulation of MAPK signalling. Specifically, in tumours bearing wild-type Raf but mutant Ras (such as pancreatic cancer), Raf inhibitors create feedback activation of MAPK signalling by inducing dimerization of cRaf with BRaf and interaction with the oncoprotein Kras-GTP. Therefore, MEK inhibition has emerged as a brighter therapeutic strategy. Additionally, to the MAPK and PI3K pathways, other Kras effectors are shown to move and functionally linked to pancreatic carcinogenesis. it'll be one among the upcoming challenges to work out the relative importance of the various Kras effectors at different stages of the disease, and in individual cases of carcinoma. Since carcinoma is related to a mutant, constitutively active sort of Kras, it's been supposed that Ras activity is consistently high in tumour cells. But it had been observed that even when mutant Kras is present in every single cell of the pancreas from the start of the organ's embryonic development, the activity of downstream effectors of Kras isn't elevated compared to the control pancreas. In point of fact, upraised activity of Kras effectors is first observed when the initial morphological alterations take place. there's a requirement for regeneration mechanisms to induce and maintain high Kras activity. as an example, ligand-driven EGFR activation is sufficient to activate
Kras signalling and is required for pancreatic carcinogenesis a minimum of during the initial stages. Other such mechanisms include inflammatory stimuli. Kras may be a key oncogene during the onset of carcinoma, and it's still required-at least during a subset of tumours-in invasive mouse and human carcinoma.

\section{Multiple mutations within individual oncogenes}

Numerous current large-scale genetic studies have reported that plentiful patients are affected by "multiple mutations (MM) within distinct oncogenes," demonstrating that MM synergistically stimulate the mutated oncogenes. $\mathrm{MM}$ are unlike single hotspot mutations with respect to mutational pattern, function, and sensitivity to molecularly targeted therapy. A recent remarkable advance in this field is the comprehensive characterization of oncogenic MM from a pan-cancer genetic study of more than 60000 tumours, showing that approximately one-quarter of oncogenes examined were significantly affected by MM. This study also identified several oncogenes showing significant enrichment of MM in a specific cancer type, such as NOTCH1 in T-cell acute lymphoblastic leukaemia and CARD11 in nonHodgkin's lymphoma. These MM are recurrently observed in main untreated tumours, signifying that they are "de novo" MM unconnected to cancer therapy. These de novo MM have more than a few genetic characteristics like mostly present in cis with concurring variant allele frequencies. $\mathrm{MM}$ in cis are sporadically observed in TSG. Both of the mutations in each $\mathrm{MM}$ are under positive selection equal to the selection on single hotspot mutations, inferring that $\mathrm{MM}$ are an amalgamation of driver-driver mutations. Third, the mutational patterns of MM are skewed from single hotspot mutations with respect to type, position and amino acid change. MM arise from a pair of mutations that are not common as single mutations. De novo $\mathrm{MM}$ are dissimilar from single hotspot mutations with respect to mutation distribution. Minor or infrequent mutations are preferentially 
selected in de novo MM, which are functionally weak individually but display stronger oncogenic potential in combination. This gives an explanation as to why functionally weak minor mutations and VUS accumulate in cancer. Moreover, this suggests the importance of epistasis in cancer. Recurrent MM are under positive epistasis, but the study of de novo MM also suggests the existence of negative epistasis and higher-order epistasis.

\section{Depicting the role of CCDC6, the first RET partner} gene in papillary thyroid carcinoma in regulating its function on CREB1 transcriptional activity: Sumoylation study

CCDC6, a $65 \mathrm{kDa}$ pro-apoptotic phosphoprotein is identified in chimeric genes as a significant repressor of the CREB1-dependent transcription. It is found that Forskolin induced posttranslational modifications by SUMO restrict the CCDC6 in the cytosol thus, limiting its interaction with CREB1.

In a variety of both thyroid and non-thyroid tumours, CCDC6 is re-arranged and CCDC6-RET fusions found lead to the formation of RET/PTCs which are chimeric genes formed by the RET tyrosine kinase with the 5' terminal region of other genes with RET/PTC1 and RET/PTC3 being the most prevalent. It is hypothesised that the CCDC6 product, H4(D10S170) plays the role of a tumour suppressor and that its inhibition could lead to carcinogenesis. Moreover, the interaction of CCDC6 with CREB1 leads to the suppression of the latter by its dephosphorylation. Sumoylation, the covalent binding of small ubiquitin-like modifiers (SUMO) peptide to lysine residues of targets along with its mammal paralogs, SUMO-1, SUMO-2 and SUMO-3 with the latter two being more prevalent has risen in importance due to its very unique biological functions with the most important being its role in transcriptional control.

Anti-CCDC6, anti-CREB1, anti-Tubulin, anti-FLAG, anti-FLAG M2, anti-SUMO1 and anti-SUMO 2/3 were among the antibodies procured for the study. PC
C13 cells, HeLa cells, B-CPAP cells and HEK293 cells were maintained in Coon's modified Ham's F12 medium, RPMI and Dulbecco's modified eagle's medium respectively. Quick-change-site directed mutagenesis system was used to generate CCDC5 wild type and CCDC6 Triple Mutant KKK74, 266, 424RRR (TM). B-CPAP and He1a and HEK293 cells were transfected with Fugene Reagent and Lipofectamin Plus respectively.

\section{Assays and tests carried out:}

In-vitro sumoylation assays were carried out making use of whole cell lysates, recombinant CCDC6 or SUMO1 and SUMO2.Nickel affinity pull down assay was used to purify the His-SUMO1-CCDC6 or HisSUMO2-CCDC6 conjugates wherein anti-CCDC6 antibody was used to detect the protein. FRET was performed by making use of the acceptor photobleaching technique on HeLa YFP-SUMO2 cells transfected by pECFP-CCDC6wt or pECFPCCDC6TM to measure the intensity of CFP fluorescence before and after YFP photobleaching along with indirect immunofluorescence. Cells washed in PBS were used in sumoylation assays following which they were analysed using Western blot, immunoblotting and immunoprecipitation. Cytoplasmic and nuclear proteins were lysed and extracted by making use of Thermo Scientific NEPER Nuclear and Cytoplasmic Extraction Kit.

Luciferase transactivation assays and ChIP experiments were performed on HEK293 cells following which qRT-PCR was performed. The data collected was studied using Student's t-test.The sumoylation assay performed to check the posttranscriptional modification of CCDC6 by SUMO proteins and showed that CCDC6 modified to give higher-molecular weight CCDC6 conjugates. Once the modification of CCDC6 by SUMO was confirmed, the modification sites were identified at three motifs: LK266NE, LK74IE, FK424RP. Point mutations that convert Lysine to Arginine are introduced to 
understand which lysine was involved in sumoylation and the data collected is confirmed using fluorescence resonance energy transfer (FRET) which shows an increase in CFP emission following YFP bleaching thus, showing the nearness of SUMO2 and CCDC6 which allows energy transfer but no FRET was found between YFP-SUMO2 and CCDC6 Triple Mutant fused to CFP. YFP-SUMO2 was found to impact the subcellular localisation of CCDC6 and in its presence the CCDC6 product wt was found in cytosolic fraction. A reduction of the ability of anti-CCDC6 antibodies to precipitate the AREG promoter region, a CREB1 target is seen in the presence of SUMO2 in BCPAP. The luciferase reporter assay showed a reduction of CCDC6-repressive function on CREB-1 transcriptional activity in the presence of SUMO2. Quantitative PCR depicted the strong inhibition of CREB1 dependent expression of transcriptional targets by CCDC6. Forskolin treatment was used to exhibit CCDC6 sumoylation dependence from cAMP activity.

The study shed light on the fine-tuning mechanism that CCDC6 plays in cell proliferation and differentiation. It highlighted the localisation of CCDC6 in the cytosol from the nucleus which was also cemented by the relocalisation of the protein to the cytosol. The dependence of CCDC6 on cAMP to activate CREB1 transcription in normal and transformed cells which could be useful in the development of newer and novel therapeutic approaches to modulate cAMP activity in the treatment of cancer.1

\section{Role of proto-oncogene SET in promoting cellular spreading and migration via cytoplasmic targeting}

The study builds on the previous studies that depicted the movement of nucleus located SET to the cytosol upon its binding to Rac1.It goes on to show that mutation of SET to SET(DNLS) leads to cell spreading and motility.
GTPase Rac1, a member of the family of RHoGTPases, acts as a molecular switch modulates cell protrusion and actin polymerisation which are the driving forces of polarised cell motility. Rac1 requires a C-terminal lipid anchor along with an adjacent polybasic region for cell migration and it binds to GEF $\beta$-PIX, Caveolin1, PACSIN2, ubiquitin ligase Nedd4, mTOR and SET/I2PPA, a proto-oncogene and inhibitor of PP2A involved in regulation of apoptosis, cell cycle and cell motility. Upon phosphorylation by Casein Kinase II the dimerization, localisation and function of SET is monitored. SET and active Rac1 present in nucleus can migrate to cytoplasm which depicts their role in cell motility and cell spreading.

Anti-SET/I2PP2A, GFP, $\beta$-catenin, ZO-1 were among the antibodies used and F-Actin was spotted using Texas-Red or Alexa-Flour-633-Labeled phalloidin. ECIS was used to analyse cell spreading in HeLa cells which were transfected using TransIT-LT1 Transfection Reagent after normalising the results to cells expressing YFP. Site-directed mutagenesis was performed on YFP-SET to form its mutants A9 and E9. A protein transduction domain was encoded following which 10 amino acids $\mathrm{N}$-terminal to the CAAX domain of Rac1 were also formed. Cell lysates were used to perform a peptide pull-down assay. Fluorescent imaging was carried out on cells transfected with indicated plasmids, seeded to fibronectin-coated glass coverslips. Nanopro 1000 System was used to quantify total levels and phosphorylation of ERK in cell lysates. A constitutively cytoplasmic mutant of YFP-tagged SET still bound to the Rac1 C-terminal peptide is formed to check the efficiency of binding of Rac1 C-terminus to full-length YFP-SET and YFP-SET mutant. Increased Rac1 activity is not found to alter YFP-SET or YFP-SET (DNLS) mutant interaction with Rac1 Cterminus. Impact of cytoplasmic targeting of SET on distribution of cytoskeletal proteins was studied using confocal imaging on transfected HeLa cells and it is found that YFP-SET (DNLS) while localised to the 
peripheral membrane ruffles did not affect the distribution of $\mathrm{F}$-actin, tubulin or vinculin. The cytoplasmic migration of SET is found to promote cell migration although it is not accompanied by clear membrane association in case of the wild type SET. Phase contrast microscopy was used to measure the wound healing responses which depicted that the cells expressing YFP-SET(DNLS)-mutant closed wounds faster than cells expressing wt SET, YFP or no construct at all.Wild type SET reduce cell spreading whereas YFP-SET(DNLS) promotes cell spreading. E9 mutant of SET shows high cytoplasmic localisation in comparison to A9 mutant which is completely nuclear. Membrane ruffles are analysed using kymograph which showed a higher number and frequency of membrane ruffles in the mutant cells as compared to wild type cells. Increasing doses of okadaic acid which is a PP2A inhibitor showed that PP2A inhibition is not involved in cell spreading and migration by cytoplasmic SET. The Nanopro assay used to study the impact on other signaling pathways shows very little reduction in phosphorylated ERK1 by expression of wild type and mutant YFP-SET while the amount of unphosphorylated ERK1 and ERK2 was found to be elevated.

SET accumulation, induced by Rac1 leading to the restoring Rac1-mediated cell motility. Cell migration speed was studied in the form of wound healing assay and cell spreading, membrane ruffling and intracellular signaling were analysed to determine the dependence of cellular responses on cytoplasmic targeting of SET. SET(DNLS) was found to not only promote wound healing but also cell spreading and increased intercellular contact which was known to be actin polymerisation. This study shed light on the wound healing properties of the proto-oncogene SET which would open up avenues for further research to develop new therapeutic approaches.
Role of polymorphisms G691S/S904S of RET protooncogene in modifying the phenotype of Medullary Thyroid Carcinoma

The aim is to study the frequency of G691S/S904S haplotype in patients with Medullary Thyroid Carcinoma (MTC) and their relatives by extracting peripheral blood leucocytes to show that the haplotypes were in complete disequilibrium. As is well known, Medullary thyroid carcinoma (MTC), a rare, autosomal dominantly inherited neoplasm of the calcitonin secreting parafollicular cells of the thyroid gland has three clinical forms: Multiple Endocrine Neoplasia type 2A (MEN2A), Multiple Endocrine Neoplasia type 2B(MEN2B) and Familial MTC with each of these forms having their own distinct features. RET proto-oncogene located on chromosome 10q11.2 containing 21 exons plays an important role in the proliferation and differentiation of cells encodes a tyrosine kinase receptor and has three domains: a large extracellular domain with a cysteine-rich region and cadherin homology domains, a transmembrane domain, and an intracellular tyrosine kinase domain. There are one of the six-point mutations observed in cysteine residues in exon10, exon11, exon13, exon15, exon16. The risk of development of sporadic MTC was found to be associated with the G691S and S904S single nucleotide polymorphisms in exon11 and exon 15 respectively.

The study included one hundred ninety MTC patients who were diagnosed with MTC after studying the histopathology of biopsy following which a questionnaire was used for information collection. Upon finding any mutation the relatives were invited as well which resulted in the participation of one hundred twenty-one relatives in the study. The DNA was extracted from the blood leucocytes and was quantitated on spectrophotometer. Polymerase chain reaction was used in order to identify the RET variant 691, 904 following which the amplified products were electrophoresed on $8 \%$ polyacrylamide gel and direct DNA sequencing method was used to confirm the presence of mutations. Chromas 2.33 was used to 
analyse the mutations and SNPs and statistical analysis was performed using SPSS 20.

A total of 293 members consisting of 181 Iranian MTC patients and 112 relatives including 33 FMTC, 6 MEN2A, 2 MEN2B, 1 pheochromocytoma and 145 apparently sMTC cases. 2 germline polymorphisms of RET gene found at codon 691 and codon 904 do not lead to amino acid alteration. The patients and relatives showed similar allele frequencies of each of these SNPs. Since, G691S and S904S variants are in complete linkage disequilibrium the results were grouped together. 57.45\% MTC patients and 49.1\% relatives exhibited the G691S/S904S gene haplotype.

82 MTCs and 47 relatives were found to be heterozygous and 22 MTCs and 8 relatives were found to be homozygous. The higher proportion of females and the age of diagnosis were not found to be significantly correlated to the presence of G691S/S904S haplotype. The cosegregation together of the two variants shows the linkage disequilibrium and the study depicted the association of both G691S and S904S SNPs with sporadic MTC and MEN2A.The study showed a higher frequency of G691S in patients with MTC and among the general population it was more in people with greater risk of cancer.The data suggested that the polymorphism enhances the oncogenecity of RET by creating new splicing sites, synthesis of truncated proteins, erroneous ligand binding, change of structure among others. The frequency of this haplotype and its modifying action on the phenotype of MTC would be a new avenue for further research. This study was particularly important since it was already well-known how important RET gene mutations were in patient management, more particularly in decision making regarding the need for total or partial thyroidectomy or the age at which the child should be subjected to surgery.

\section{Evaluating the role of the proto-oncogene DEK in the development and progression of astrocytic tumours}

One of the highest rates of relapse post-surgery are seen in astrocytic tumours which is why early diagnosis is of utmost importance which is aided by developing an understanding of the molecular mechanism for tumorigenesis and progression for astrocytic tumours. Upon quantification of DEK mRNA and protein expression in normal and astrocytic brain tissues it was found that DEK was highly expressed in astrocytic tissues with a positive relation between its expression and grade of the tumour. This study strongly points towards the role of DEK in proliferation and apoptosis as observed in U251 glioblastoma cells.

The highly infiltrative nature of astrocytic tumours and along with an increased incidence of grade III and IV tumours make complete surgical removal of the tumour very difficult leading toa need for alternative routes of therapy thus, gene therapy involving the study of genes responsible for the formation, development and prognosis of tumour was considered as a potential therapeutic target. Among such genes is the DEK proto-oncogene, preferentially expressed in actively proliferating and malignant cells allowing them to play an important role in tumour formation by inhibition of cellular senescence and differentiation, facilitation of cell transformation, and the prevention of apoptosis. An overexpression of DEK is well-linked with tumour growth, late pathological stage, and poor prognosis.

The tissue specimens were obtained over a span of 2 years, a total of 32 astrocytic tumour specimen were obtained by neurological excision and normal brain tissues were obtained from patients with craniocerebral trauma by decompression surgery and within this, 19 were male and 18 were female specimens. Out of the 32 specimens, 14 were lowgrade and 18 were high-grade. The human 
glioblastoma cells obtained were maintained in Dulbecco's modified Eagle's medium supplemented with $10 \%(\mathrm{v} / \mathrm{v})$ fetal bovine serum at 37 degree Celsius with $5 \% \mathrm{CO}_{2}$. From the cells, RNA was isolated using TRIZol reagent and reverse transcribed using the polymerase chain reaction following which the separated and stained amplified products were studied for their fluorescence and integrated density value (IDV) for DEK and $\beta$-actin genes. The IDV ratios for DEK and $\beta$-actin were used to depict the relative DEK mRNA expression. The U251 cells transfected with small interfering RNA (siRNA) using Lipofectamine 2000 were seeded on cell culture plates after which they were separated into three groups: the siRNADEK group, the negative control group with nontargeting siRNA and the normal control group which had no siRNA. Cells were collected after $48 \mathrm{~h}$ for downstream analysis for which Western blotting was used to visualise immunoreactive proteins. The immunohistochemistry was studied by deparaffinising the paraffin embedded sections rehydrating them in ethanol and hydrating it with water and phosphatebuffered saline (PBS) which were then incubated with streptavidin-biotin complex (SABC) and stained with hematoxylin. The staining intensity of cells was scored as: 0 for no staining, 1 for light brown; 2 for brown and 3 for dark brown. Upon counting 200 cells, five random fields were selected to understand the proportion of DEK positive cells as 0 for $<5 \%, 1$ for 5 $25 \%, 2$ for $26-50 \%, 3$ for $51-75 \%$ and 4 for $>75 \%$.Using the staining intensity and proportion the DEK expression was calculated and the values assigned were: 0-1 for negative (-), 2-5 for weak positive expression $(+), 6-8$ for positive $(++)$ and $>9$ for strong positive $(+++)$ expression Image analysis was captured by BX51 microscope and optical density average, integral optical density average and percentage of positive cells were calculated. Cell proliferation assay was performed by collecting cells transfected with siRNA at 24, 48 and $72 \mathrm{~h}$ and incubating them with serum-free optimMEM medium and 3-(4,5-dimethylthiazol-2-yl)-2,5- diphenltetrazolium bromide (MTT) with the data being represented as mean standard deviation (SD). Apoptosis assay and cell cycle measurement were both quantified by flow cytometry. Upon lysing the cells with cold lysis buffer, incubating them on ice and mixing them with reaction buffer and enzyme substrate the absorbance was measured at $405 \mathrm{~nm}$ with a microplate reader to measure the Caspase- 3 activity All the data collected was analysed using SPSS and one-way analysis of variance (ANOVA) was used to test the statistical significance. Upon reverse transcription PCR, DEK mRNA was detected in 30 out of 32 patients (93.8\%) with astrocytic tumours and in 1 out of 5 patients (20\%) with cerebral hemorrhage. The ratio of DEK IDV $/ \beta$-actin IDV was found to be $0.30 \pm 0.18$ in patients with low-grade astrocytic tumours and $\mathrm{d} 0.80 \pm 0.10$ in patients with high grade astrocytic tumours. Western blotting showed DEK positive in $96.88 \%$ in tumour cases and in $40 \%$ in normal cases. Overall, $88.3 \% \pm 5.6 \%$ of cells in the high-grade tumors, $51.6 \% \pm 9.7 \%$ cells in the lowgrade tumors, and $36.0 \% \pm 5.7 \%$ cells in the normal brain tissues were found to be DEK positive. The DEK mRNA level was found to be higher in the negative control and blank control groups than the siRNADEK-transfected group. After siRNA treatment of U251 cells the DEK protein expression was found to reduce significantly and the impact of depletion of DEK on cell morphology exhibited by the increased cell debris, reduced transparency with a lot of particles and vacuoles in the DEK siRNA-treated cells. The cell optical density was found to be $0.70 \pm 0.06$ for the siRNA-DEK, $0.83 \pm 0.08$ for the negative control, and $0.87 \pm 0.07$ for the blank control group and similarly, flow cytometry was used to analyse cell cycle which showed that the number of cells in $\mathrm{S}$ phase was reduced and the fraction of cells in G0/G1 and $\mathrm{G} 2 / \mathrm{M}$ phases were increased in the siRNA-DEK group. Cell apoptosis was found to be significantly lower after siRNA treatment with no marked difference found in negative and blank control. The level of p53 and p21 proteins were found to be 
$0.87 \pm 0.06$ and $0.86 \pm 0.09$, respectively in the siRNADEK group, which was significantly higher than the proteins in the negative control $(0.46 \pm 0.07$ for $\mathrm{p} 53$ and $0.58 \pm 0.07$ for p21) and the blank $(0.48 \pm 0.09$ for p53 and $0.55 \pm 0.06$ for p21) groups. Caspase-3, an enzyme activated by proteolytic cleavage during apoptosis was found to have increased activity in the DEK-depleted cells. The previous studies on DEK studied its role in many tumours but its role in astrocyctic tumours was less known thus, this study provided new therapeutic targets which would lead to invention of new therapies that could be used to overcome this lethal disease. The results highlighted the tumour cell death caused due to depletion of DEK expression. This study provided sufficient evidence that DEK could be a potential target gene for the diagnosis and therapy of astrocytic tumours. The paper highlights the various factors such as DEK upregulation, inhibition of p53 and cell cycle progression and reduction of cell apoptosis due to inhibition of Caspase-3 which leads to tumour formation. 4

\section{Action of c-Kit as a dependence receptor: tumour inhibition}

Previous studies conducted on c-Kit leave no uncertainty about its proto-oncogenic role thus, explaining its constitutive kinase action causing out of control proliferation. But, some of the actions of c-Kit cannot be attributed to its proto-oncogenic activity alone and this paper depicts the active role c-Kit plays in cell apoptosis unless attached by stem cell factor (SCF). Cleavage at D816 by protease enzyme is involved in the tumour suppressive action of c-Kit. Dependence receptors are the type of receptors which can transduce intracellular signals even in the absence of their ligands thus, they can trigger the induction of apoptosis making the cells dependent on the availability of ligand for their survival. c-Kit or CD117, a viral oncogene coding for a type III receptor tyrosine kinase (RTK) plays an important role in cell proliferation, differentiation and migration by binding with its ligand the stem cell factor (SCF) protein after which it undergoes an autophosphorylation cascade. In many cases of cancer, the dysregulation of c-Kit is related to tumorigenesis but in certain cancers such as neuroblastoma the expression of c-Kit reduces which is not a regular trait of proto-oncogene but that of a dependence receptor.

Several stable cell lines were generated in which doxycycline was used to induce c-Kit expression and the inactivation of c-Kit kinase activity was verified by evaluating c-Kit auto-phosphorylation as well as ERK $1 \frac{1}{2}$ phosphorylation.

In A549 cell line it was found that the overexpression of wild type c-Kit was not sufficient to induce cell death as compared to the kinase dead c-Kit which massively enhanced cell death. It was also found that an overexpression of c-Kit strongly induces caspase activity. It was also found that ligand SCF is able to revert apoptosis induced by c-Kit. Caspases are one of the key components of the death of dependence receptors thus, the role of caspase cleavage in c-Kit induced apoptosis was studied using double mutated stable cell lines which dramatically altered the proapoptotic activity of kinase mutated c-Kit. The relationship between caspase cleavage of c-Kit and caspase-9 recruitment and activation was studied and it was found that there was no interaction between mutated c-Kit and caspase- 9 .

To show the intrinsic tumour suppressive activity HMCB cells, inducible for kinase-mutated c-Kit were ingrafted in severe combined immunodeficiency (SCID) mice and the tumours were treated by intraperitoneal injections of doxycycline (or vehicle) to induce c-Kit expression which led to strong and prolonged tumour inhibition. It was found that mutation of the caspase site was successful in preventing the tumour suppressor activity of c-Kit. 
Next, the blocking interaction of SCF with c-Kit was studied by silencing the ligand using RNAi strategy in various cancer cells which showed massive enhancement of cell death induction upon SCF silencing. A stable HCT116 cell line inducible for the expression of the extracellular domain of c-Kit was generated and it was found that overexpression of cKit-EC on treating with doxycycline triggered apoptotic cell death. c-Kit RNAi strategy was used to show that the cell death triggered by interference with SCF and c-Kit binding was due to the proapoptotic activity of c-Kit rather than an indirect effect of switching off the survival kinase-dependent signal.

All existing studies are solely focused on the kinase activity of the proto-oncogene leading to development of therapeutic approaches targeting the inhibition of its kinase activity E.g., Imatinib, a drug effective in many cancers leads to resistance in majority of the patients causing c-Kit to remain active. This study focuses on the alternative pathway of inhibiting c-Kit kinase activity which would target interaction between SCF and c-Kit to both inhibit the kinase activity and to induce c-Kit-induced cell death. The study thus, provided an alternate route of treatment for cancers.

\section{IV.CONCLUSION}

This research review's purpose is to help the reader understand different aspects posed by the research on the relationship of oncogenes proto-oncogenes. This is significant because it gives insights about both oncogenes and proto-oncogenes. There has been much research and discussion conducted on these opinions on the relationship between these two. Most of the research found was on the Keywords. More research and testing is required to gain a better understanding of this relationship.

\section{ETHICS APPROVAL AND CONSENT TO PARTICIPATE.}

Not applicable.

\section{HUMAN AND ANIMAL RIGHTS}

No Animals/Humans were used for studies that are base of this research.

\section{CONSENT FOR PUBLICATION}

Not applicable.

\section{AVAILABILITY OF DATA AND MATERIALS}

The author confirms that the data supporting the findings of this research are available within the article.

\section{FUNDING ACKNOWLEDGEMENT CONFLICT OF INTEREST}

AND

The authors whose names are listed immediately above certify that they have NO affiliations with or involvement in any organization or entity with any financial interest (such as honoraria; educational grants; participation in speakers' bureaus; membership, employment, consultancies, stock ownership, or other equity interest; and expert testimony or patent-licensing arrangements), or nonfinancial interest (such as personal or professional relationships, affiliations, knowledge or beliefs) in the subject matter or materials discussed in this manuscript.

\section{REFERENCES}

[1]. Alitalo, K. Amplification of cellular oncogenes in cancer cells. Trends Biochem. Sci. 10, 194197 (1985).

[2]. Stowers, S. J., Maronpot, R. R., Reynolds, S. H. \& Anderson, M. W. The Role of Oncogenes in Chemical Carcinogenesis. Environ. Health Perspect. 75, (1987). 
[3]. Weber, J. \& Mcclure, M. Regular Review Oncogenes and cancer. Br. Med. J. (Clin. Res. Ed). 294, (1987).

[4]. Forgacs, I. Oncogenes and gastrointestinal cancer. Gut vol. 29 417-421 (1988).

[5]. Garte, S. J. \& Burns, F. J. Oncogenes and Radiation Carcinogenesis. Environ. Health Perspect. 93, (1991).

[6]. Polsky, D. \& Cordon-Cardo, C. Oncogenes in melanoma. Oncogene vol. 22 (2003).

[7]. Hammond, S. M. MicroRNAs as oncogenes. Current Opinion in Genetics and Development vol. 16 (2006).

[8]. Collins, M. A. \& di Magliano, M. P. Kras as a key oncogene and therapeutic target in pancreatic cancer. Frontiers in Physiology vol. 4 JAN (2014).

[9]. Cisowski, J. \& Bergo, M. O. What makes oncogenes mutually exclusive? Small GTPases vol. 8 (2017).

[10]. Saito, Y., Koya, J. \& Kataoka, K. Multiple mutations within individual oncogenes. Cancer Science vol. 112 (2021).

[11]. Pulciani, S. et al. Oncogenes in human tumor cell lines: Molecular cloning of a transforming gene from human bladder carcinoma cells. Proc. Natl. Acad. Sci. U. S. A. 79, (1982).

[12]. Bartram, C. R. Oncogenes: Clues to carcinogenesis. European Journal of Pediatrics vol. 141 (1984).

[13]. Busch, H. Molecular lesions in cancer. Mol. Cell. Biochem. 61, (1984).

[14]. Neckameyer, W. S., Shibuya, M., Hsu, M. T. \& Wang, L. H. Proto-oncogene c-ros codes for a molecule with structural features common to those of growth factor receptors and displays tissue specific and developmentally regulated expression. Mol. Cell. Biol. 6, (1986).

[15]. Carè, A. et al. Translocation of c-myc into the immunoglobulin heavy-chain locus in human acute B-cell leukemia. A molecular analysis. EMBO J. 5, (1986).
[16]. Barbanti-Brodano, G. et al. Induction of Malignant Subcutaneous Sarcomas in Hamsters by a Recombinant DNA Containing BK Virus Early Region and the Activated Human cHarvey-ras Oncogene. Cancer Res. 47, (1987).

[17]. Chen, C. H. et al. Recombinant DNA vaccines protect against tumors that are resistant to recombinant vaccinia vaccines containing the same gene. Gene Ther. 8, (2001).

[18]. Laderian, B., Zhou, M. \& Fojo, T. Distribution of cancer genes in human chromosomes. Seminars in Oncology vol. 47 (2020).

[19]. Luise, C. et al. Identification of Sumoylation Sites in CCDC6, the First Identified RET Partner Gene in Papillary Thyroid Carcinoma, Uncovers a Mode of Regulating CCDC6 Function on CREB1 Transcriptional Activity. PLoS One 7, (2012).

[20]. Lam, B. D., Anthony, E. C. \& Hordijk, P. L. Cytoplasmic targeting of the proto-oncogene SET promotes cell spreading and migration. FEBS Lett. 587, 111-119 (2013).

[21]. Sheikholeslami, S., Zarif Yeganeh, M. \& Hoghooghi Rad, L. Haplotype Frequency of G691S/S904S in the RET Proto-Onco-gene in Patients with Medullary Thyroid Carcinoma. Iranian J Publ Health vol. 43 http://ijph.tums.ac.ir (2014).

[22]. Feng, T., Liu, Y., Li, C., Li, Z. \& Cai, H. DEK proto-oncogene is highly expressed in astrocytic tumors and regulates glioblastoma cell proliferation and apoptosis. Tumor Biol. 39, 1-10 (2017).

[23]. Olivos, D. J. et al. The proto-oncogene function of Mdm2 in bone. J. Cell. Biochem. 119, 88308840 (2018).

[24]. Wang, H. et al. The Proto-oncogene c-Kit Inhibits Tumor Growth by Behaving as a Dependence Receptor. Mol. Cell 72, 413-425.e5 (2018).

[25]. Silva, T. M. et al. Triiodothyronine (T3) upregulates the expression of proto-oncogene 
TGFA independent of MAPK/ERK pathway activation in the human breast adenocarcinoma cell line, MCF7. Arch. Endocrinol. Metab. 63, 142-147 (2019).

[26]. Pedersen, K. B. et al. Liver tumorigenesis is promoted by a high saturated fat diet specifically in male mice and is associated with hepatic expression of the proto-oncogene Agap2 and enrichment of the intestinal microbiome with Coprococcus. Carcinogenesis 40, 349-359 (2019).

[27]. Eadyow, K., Phusantisampan, T., Maneechay, W. \& Sangkhathat, S. Genetic polymorphisms of the SOX10 gene in Thai patients with sporadic Hirschsprung disease. / Songklanakarin J. Sci. Technol vol. 42.

[28]. Sara, M., 1, D., Tina, Ž. \& Barbara, P. Ret protooncogene mutations in the Slovenian population of patients with medullary thyroid cancer. doi:10.25670/oi2020-002on.

\section{Cite this article as :}

Nehal Batra, Ishita Ghag, Karishma Babu, Tejaswini Divanji, " Reviewing Oncogenes and ProtoOncogenes ", International Journal of Scientific Research in Science and Technology(IJSRST), Print ISSN : 2395-6011, Online ISSN : 2395-602X,Volume 8, Issue 3, pp.458-479, May-June-2021. Available at doi $\quad:$ https://doi.org/10.32628/IJSRST2183100

Journal URL : https://ijsrst.com/IJSRST2183100 